\title{
A new method for surface crack detection by laser thermography based on Thermal Barrier effect
}

\author{
By Pablo López de Uralde*, Eider Gorostegui-Colinas*, Ander Muniategui*, Izaskun Gorosmendi*,
} Borja Hériz*, Mikel Ayuso*, Xabier Sabalza* *IK4-LORTEK, Control and Evaluation Department, Arranomendia Kalea 4A, 20240, Ordizia, Gipuzkoa, Spain,
egorostegui@lortek.es

\begin{abstract}
The aim of this work is to detect open surface cracks on metallic welds using laser thermography and posterior processing algorithms. In the last years the trend on welding processes has been to extend their use to applications with higher stress conditions. As a consequence, obtained welded regions are more prone to cracks. The types of cracks originated from welding processes are, in general, superficial and their detection is not straightforward since it detection is affected by their width.

Traditional crack detection Non Destructive Techniques, such as Dye Penetrant or Magnetic Particles, are time consuming and require an experienced technician to correctly interpret the results. Furthermore, they foul the surface which needs to be cleaned after the test. Laser Thermography [5] [10] has several advantages over these traditional techniques: it is fast, non-contact and clean.

In this paper, dynamic tests have been performed by scanning the weld maintaining the laser and camera motionless, and moving the piece linearly across them, so that the weld is scanned. The use of a scanning laser line to heat the surface allows the detection of the characteristic disruption that cracks generate in the natural diffusion of heat on the surface. Briefly, cracks block the heat front. This makes the temperature just before the crack to increase, while keeping the temperature of the region just after it at similar values for larger times than for other nearby regions. This thermal effect is known as "Thermal barrier" [11]. Here, a new method to detect the cracks based on this effect has been developed starting from two different approaches.
\end{abstract}

\section{Introduction}

The trend of the last years has been subjected to the application of welded joints to higher stress conditions. This situation makes the welded region to be more prone to cracks. In general, cracks that originate during the welding process are superficial and their detection is affected considerably by their width. There are different Non Destructive Testing methods commonly used to detect surface cracks, such as Penetrant Testing, Magnetic Particles and Eddy Currents. Penetrant Testing and Magnetic Particles are manual methods that require time and experienced technician to correctly interpret the results. Furthermore, they foul the surface and as a consequence it needs to be cleaned after each test.

Thermography is an emerging NDT method that has several advantages as it is fast, non-contact, and provides full field of information to be post-processed. Flash Thermography and Lock-In Thermography are commonly used to detect in-depth defects parallel to the surface, but are not always appropriate to detect surface cracks in metals due to their high conductivity. As for Induction Thermography, it is based on the detection of Eddy Currents and it requires the coil to be placed close to the surface of the material to inspect. Due to the characteristics of the cracks and the impossibility to access to the weld in two of the analyzed specimens in this paper, laser thermography[4] [9] has been selected for crack inspection in welded components[3].

Using a scanning laser line to heat the surface allows the detection of the characteristic disruption that cracks generate in the natural diffusion of heat in the surface. Briefly, cracks block the heat front, which makes the temperature just before the crack to increase, while the region just after it keeps its temperature more time than other nearby regions due to the heat conduction alteration provoked by the crack [2]. This thermal effect is known as "Thermal barrier" [8] and a new method to detect it has been developed.

The method has been firstly applied to the dynamic sequence, where laser and camera remain static and the piece is moving on a linear motion table. It has also been applied to a recording previously manipulated in order to reconstruct it. The reconstruction method employed is also described in this work. Briefly, the main idea behind the reconstruction is to convert the original dynamic measurement to a sequence in which the sample is static while the laser scans the sample. 
To summarize, the developed crack detection algorithm has been applied to measurements that come from two different approaches, being the main objective of this work to determine which procedure gives the best crack detection results.

\section{Experimental Measurements}

\subsection{Details of the inspected samples}

Two different types of cracks (see Fig.1a) have been selected to test the reliability of the detection algorithm. The first group (henceforth called LMD) of welds analyzed on this work have real open surface cracks generated on a process of Laser Melting Deposition. The substratum material employed has been Stainless Steel AISI 316 and Stellite 6 has been used as powder. The cracks propagate perpendicularly to the weld and generally cross them side to side, having an approximate length of about $25 \mathrm{~mm}$ and a width of $70 \mu \mathrm{m}$.

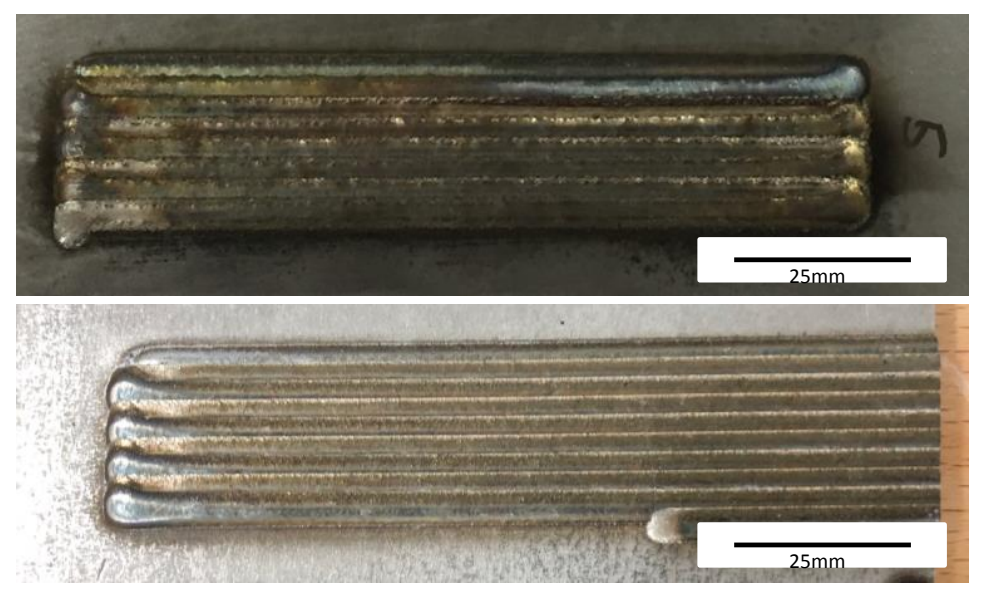

LMD 1

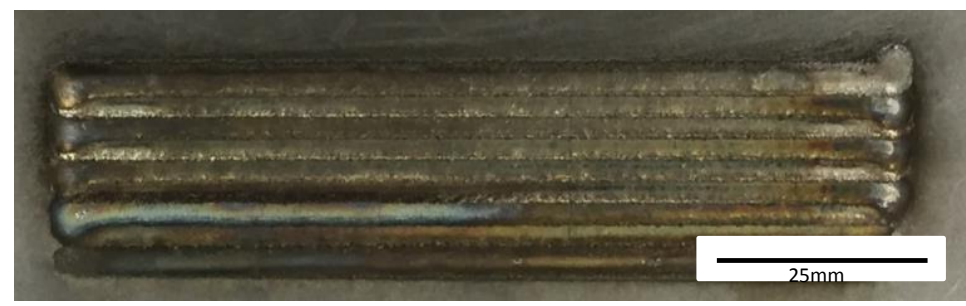

LMD 2

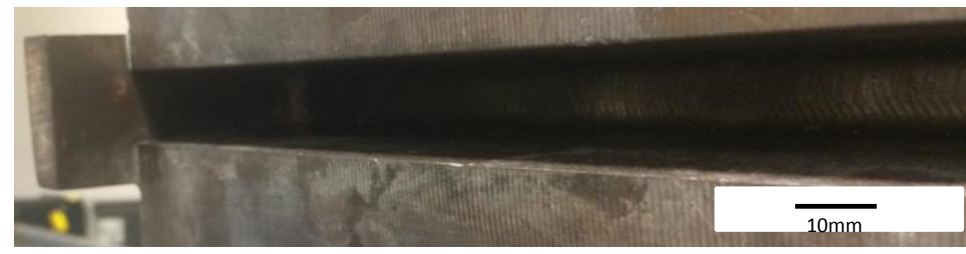

TIG 1

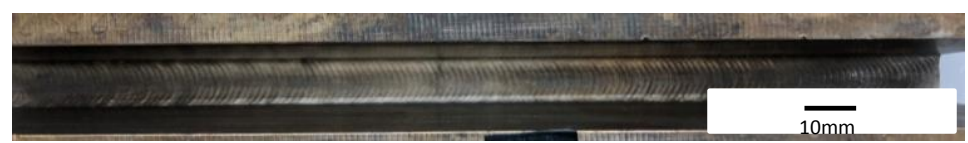

TIG 2

Fig. 1a: Set of pieces inspected in this paper, divided into two groups depending on the welding type (LMD and TIG multipass Narrow Gap Welding).

The second group (henceforth called TIG) have consisted of two real open surface cracks generated on a process of TIG multipass narrow gap welding. Characteristics of the weld are shown in Figure 1b. The material of the test samples has been AISI 316LN. The fact that the gap is extremely tight does not only affect to the detection, but also to the welding process itself. The specimen has been analyzed after the first welding process, which has implied an additional challenge to the thermographic measurement. In this case, the length of the cracks is approximately $7 \mathrm{~mm}$ and their width is about $20 \mu \mathrm{m}$. 

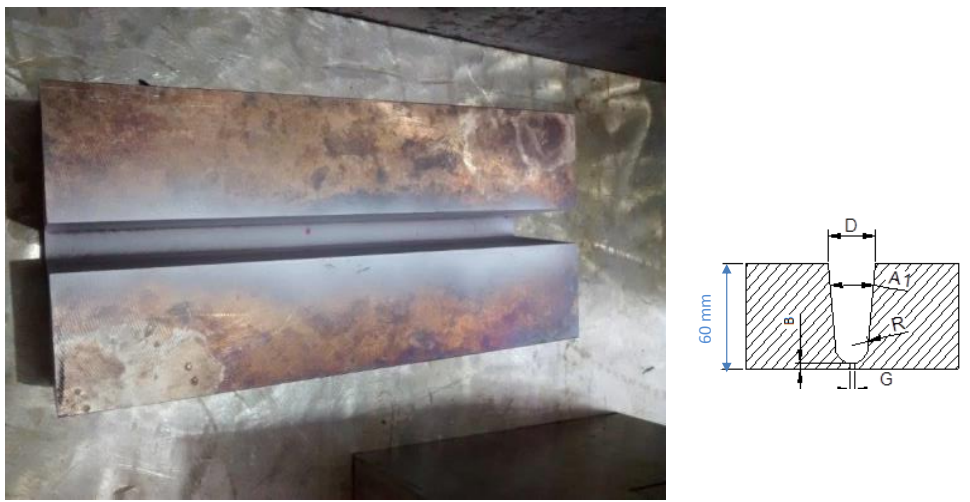

Fig. 1b: Characteristics of TIG multipass narrow gap welding.

\subsection{Set up}

One of the most important requirements of the thermography tests performed has been to get a laser line adapted specifically to the test samples. As mentioned before, LMD samples have longer cracks so the experiments have required a line able to reach both edges of the weld. In the TIG case, the most important requirement has been to create a line that could adequate to the geometry of the specimen. It means that the laser only has to affect to the welded part, avoiding any type of reflections that can ruin the measurements. This is why for this work a fiber diode laser has been selected. Starting from the end of the fiber, a plano-convex lens has been added to focus energy on a small point of $2 \mathrm{~mm}$ of diameter. Finally, to create the line, a cylindrical convergent lens has been located at the previous point location. As a result, for the LMD welds, a $25 \mathrm{~mm}$ long laser line with $3 \mathrm{~mm}$ width has been obtained, which is long enough to inspect the whole width of weld without sacrificing too much the energy density. In the second case, for the TIG welds, a smaller line has been needed, so its length was about $10 \mathrm{~mm}$.

The laser used on the measurements has been a fiber diode laser in Continuous Wave mode, with a maximum power of $50 \mathrm{~W}$ and a wavelength of $808 \mathrm{~nm}$. Due to the different nature of the cracks inspected in each group (LMD and TIG multipass) and the previous experience on these specimens, different laser powers have been used on each one: $10 \mathrm{~W}$ and $30 \mathrm{~W}$ have been selected for LMD and higher powers, 30W and 50W, for TIG.

As for the camera, a cooled thermal camera has been used to record the thermal sequences. This camera has a focal plane array InSb detector with a resolution up to $640 \times 512$ pixels, working in the short wave region at 1.5-5.5 $\mu \mathrm{m}$ and a Noise Equivalent Temperature Difference (NETD) of less than 25mK (typically 18mK). For LMD, it has been situated at a distance of $50 \mathrm{~cm}$ from the object, giving an approximately spatial resolution of $150 \mu \mathrm{m} / \mathrm{pixel}$. For TIG specimens, an extension ring has been added to the camera in order to reduce the distance between piece and camera to $15 \mathrm{~cm}$ and decrease the $\mu \mathrm{m} / \mathrm{pixel}$ ratio to 60 . Finally, the frame rate has been set to $200 \mathrm{fps}$ in all the measurements.

To generate the relative movement between laser/camera (static) and test piece (dynamic) a linear motion system has been used, setting different test speeds to evaluate its importance on the measurements. Set up is shown in Fig.2. The different number of frames derived from the different velocities has been taken into account in the detection algorithm.

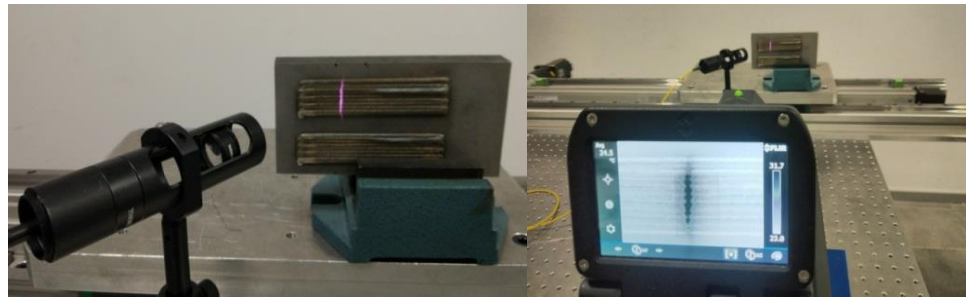

Fig. 2: Experimental set up showing the laser line and the test sample.

\subsection{Measurement description}

The experimental measurements have consisted on a linear scan of the laser line [5] [6] [1] along the weld, with the laser parallel to the direction of the cracks. Different laser powers (10-50W) and linear motion system speeds (50$400 \mathrm{~mm} / \mathrm{min}$ ) have been set to stablish the importance of these two parameters on the detection. 
As there were two different types of cracks, different measurement parameters have been needed. For LMD, higher speeds have been selected because the main characteristic of these cracks is their width. As it is big, it was expected to help the appearance of the desired "Barrier Effect" so there has been no need to check slowest speeds. The values chosen to cover a wide range of speeds and determine the optimal have been 100, 200 and $400 \mathrm{~mm} / \mathrm{min}$.

Based on previous laser experiences on these cracks and their width mentioned above, laser power has been set at 10 and $30 \mathrm{~W}$. The line created to improve the measurements productivity caused a decrease of the power density (not measured on this work).

Test pieces LMD 1 and LMD 2 have been selected to develop the whole bunch of tests including power and speeds possibilities. Once this phase is over, optimal parameters have been decided based on the results obtained from the detection algorithm, and this configuration has been confirmed by using LMD 3.

For the TIG Group, the characteristics of the cracks were quite different. Besides their smaller length, their surface width is considerable tighter. For this reason, conclusions deducted from LMD cannot be directly applied to these. Slower speeds have been tested $(50,100$ and $200 \mathrm{~mm} / \mathrm{min})$ while laser power has been slightly increased considering what explained before about power density (30 and50 W).

Unlike LMD group, only two different test pieces (TIG 1 and TIG 2) were available in this case. All the possible tests, combining the power and speed parameters, have been carried out and optimal parameters have been decided.

The described set up together with the experimental have provided an optimal heat in plane diffusion for the cracks analyzed to generate the "Thermal barrier" effect desired for the detection algorithm. Once recorded the original thermal sequence, the developed crack detection algorithm has been applied, considering the two approaches described in the following lines.

\section{Crack detection algorithm}

Crack detection algorithm has been developed based on the following assumptions: 1) the thermal barrier effect provokes a Gaussian derivative-like profile and hence its integral should be Gaussian-like (i.e. sum for discrete signals), 2 ) this profile is expected to be observed only in regions nearby the cracks, and 3) the width of this profile will depend of the experimental setup (speed and power). In order to detect the cracks the algorithm extracts the thermal profile at each point in the piece under study within a predefined time interval (Figure 3 a) Desired thermal profile), mean normalizes the signal (Figure 3 a) Normalized to zero mean) and its cumulative sum is determined (Figure 3 b) expected Gaussian derivative and its cumulative sum or integral). The algorithm retrieves for each of the point of the piece the maximum value of the cumulative sum (Figure 3 b) Maximum value).

As mentioned before, two different approaches have been used in this paper. In the first approach the raw thermal information is used to detect the cracks while in the second approach the thermal information is transformed into a static piece (moving laser) equivalent visualization. Hence, the algorithm must accommodate to each of these approaches.
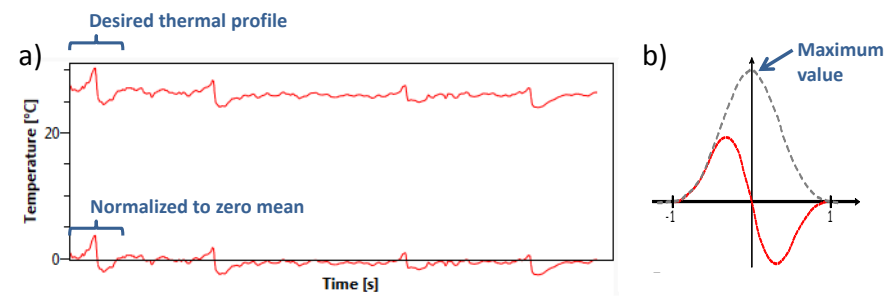

Fig. 3: a) Desired thermal profile and normalized to zero mean; b) Expected Gaussian derivative and its cumulative sum

\section{Crack detection applied directly to the dynamic thermal sequence}

The first approach considers the original thermal sequence recorded by the camera, so all the information stored in the recording has been used. In this case there is no pre-processing of the sequence, the test piece crosses the laser zone and in the camera screen only the part of the specimen affected by the laser can be seen.

Fig. 4 shows the structure of the raw thermal matrix ( $d 1$ and $d 2=$ dimension of the cameras field of view along d3 = time). Hence, 2D images of size (d1, d2) correspond to a static laser with a moving piece in the background. For a given temporal window in $\mathrm{d} 3$, at each pixel in the 2D image the thermal effect due to the movement of the piece along the cameras field of view is observed. If in this temporal window a crack moves from left to right (or the other way round) in the cameras field of view, the thermal effect (i.e. thermal barrier effect) will be observed at those pixels nearby the 
laser region, i.e. a Gaussian derivative distribution will be detected in those pixels. Hence, the algorithm must be run for each of the pixels in the $2 \mathrm{D}$ image using a sliding window of predefined size along the $\mathrm{d} 3$ dimension.

The use of this raw thermal sequence has some advantages. One of them is that it has all the information recorded and the final results provide a more accurate image, what helps to the final purpose of locating the detected cracks. On the other side, one of the biggest disadvantages is due to the same characteristic; having that amount of information leads to longer times of analysis.

\section{Crack detection applied to the reconstructed thermal sequence}

Keeping in mind the objective of obtaining good quality images in the shortest possible time, a second approach has been developed to reduce the total amount of information needing for the processing and create an easier display of sequences. This is explained on the next paragraphs [7].

Raw thermal sequence (see Fig. 4) has been recorded by infrared camera. Here we have all the information available but there is a relative movement between camera (blue rectangle represents the field of view) and laser (static red line on last shown frame) on one side and piece (green) on the other. This can lead to difficulties when trying to analyze and detect possible defects.

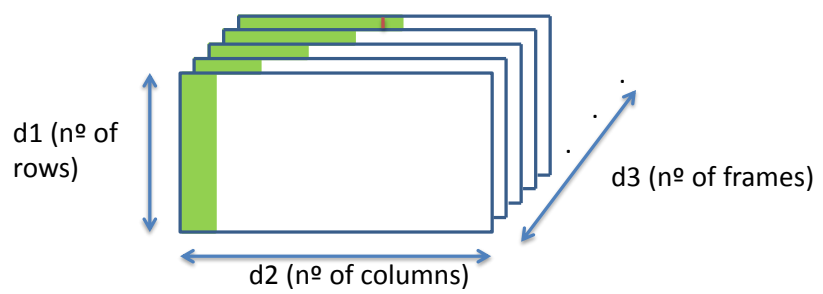

Fig. 4: Original thermal sequence diagram.

To solve this, and keeping in mind the post-processing, data has been reorganized. A static image during the whole sequence is desired, not only for analytical reasons, but also for easier display of the information. Having the whole piece always in screen makes defect location clearer.

To create this first reconstruction, columns along third dimension (time) are rearranged. The fact that the reorganization has been based on columns, leads us to a new $3 \mathrm{D}$ matrix with a third dimension equal to the second dimension ( $n^{\circ}$ of columns) of the original sequence. First dimension ( $n^{\circ}$ of rows) on both cases is the same while second dimension of the reconstruction depends on the number of frames of the original.

On this reconstruction (see Fig. 5) the whole piece is shown in every frame and when the laser turns on it will affect the entire piece at the same frame on the figure (represented as red in Fig.5) and cooling down will be homogeneous (represented as orange and green in Fig.5). This new sequence can be useful for certain type of analysis, as it transforms a dynamic test in a static one. However, the approach developed on this paper required one step more.

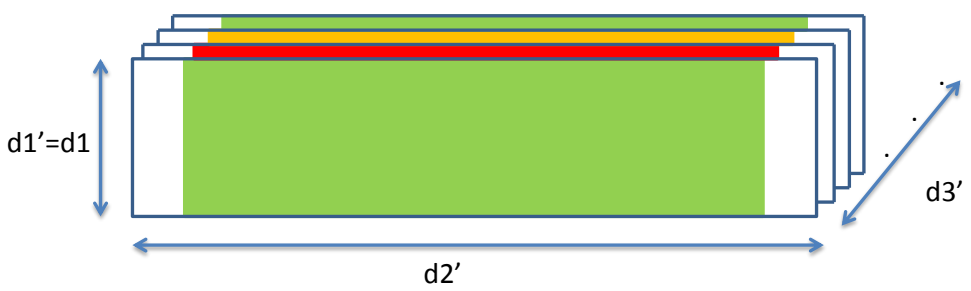

Fig. 5: Static reconstruction with laser heating homogeneously.

To create (more accurately to visualize) the Thermal Barrier effect, the laser has to move along the weld passing through the cracks. In this way, heat will accumulate on one side of the crack as the laser approximates and the other will remain still at ambient temperature (or at least at a lower temperature compared with first edge).

Based on the reconstruction previously explained, information about laser effect is added and a third sequence (second reconstruction. See Fig. 6) is generated. This last static reconstruction has all the information needed to apply the new crack detection method developed on Section 3. 


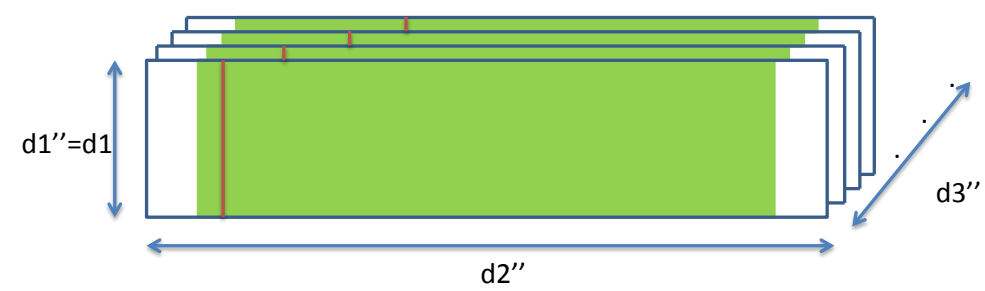

Fig. 6: Static reconstruction where laser moves through the object.

In this second approach the algorithm first reduces the obtained thermal matrix shown in fig. 5 (d1" and d2" are related with the size of the whole piece and $\mathrm{d} 3$ " is associated with the time required by the laser to move from one side to the other of the piece) to a 2D image by only taking the column pixels of the 2D image of size (d1",d2") at a predefined distance before the laser (i.e. it is as if these pixels were moving coupled with the laser). Hence, in this case the algorithm determines the maximum value of the cumulative sum of the signal extracted from the sliding window of a given size.

Both original recorded sequence and final reconstruction can be seen in Figure 7 to see the differences in the image visualization before the algorithm. Original sequence shows only the field of view of the camera and the laser static while the final reconstruction allows visualizing the entire piece and the laser moving through it.

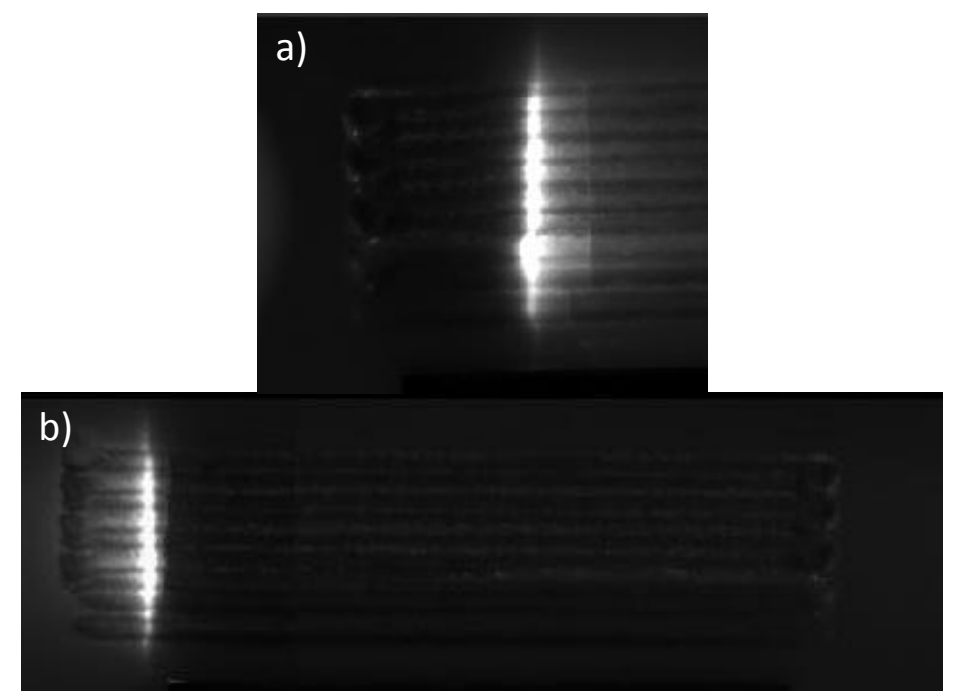

Fig. 7: Thermogram from original sequence (a) and final reconstruction (b).

\section{Results and comparison}

Before presenting the final results with the optimal set up parameters, it's important to explain the previous work that has led to that conclusion. The discussion of the selection of parameters has been realized using the first approach and, once selected the optimal, comparison between approach one and two has been done to determine the best results. It has to be mentioned that for display reasons the horizontal axe has been distorted so the images are compressed.

Firstly, test speed comparison has been developed by analyzing test pieces LMD 1 and LMD 2 at 400,200 and $100 \mathrm{~mm} / \mathrm{min}$. while maintaining the laser power at $30 \mathrm{~W}$. Some results of the detection algorithm applied to these two original sequences (Approach 1) are shown in Table1. 


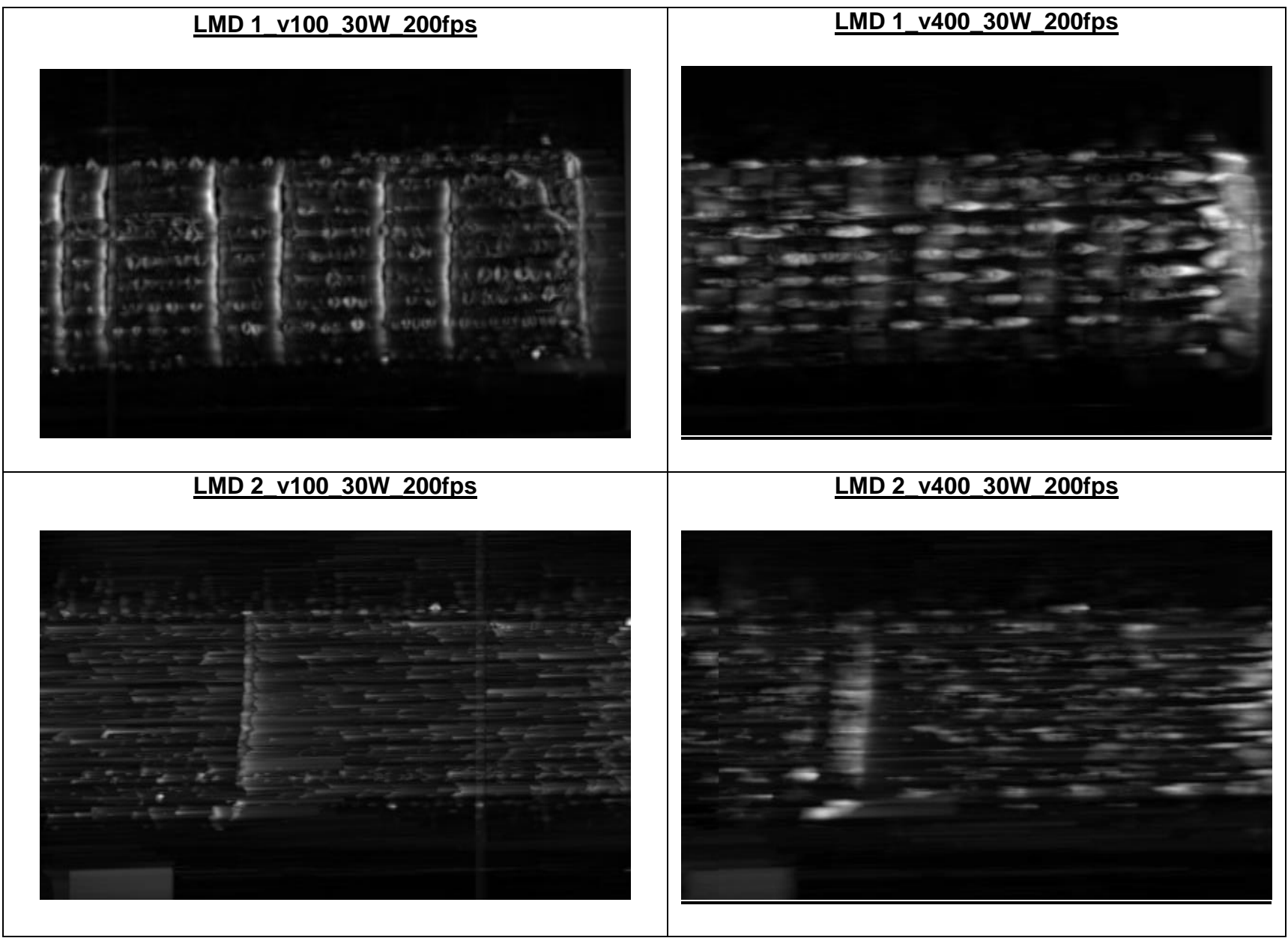

Table 1. Optimal set up parameters discussion focused on speed variations (LMD).

As can be seen, increasing the test speed has negative effect on the crack detection. Not only the images have more noise but the crack itself generates a slighter signal. The conclusion adopted is that $100 \mathrm{~mm} / \mathrm{min}$. yields the best detection results. Once fixed the test speed, laser power needs to be determined and, for that purpose, comparison between 10 and 30W are shown in Table2 (maintaining speed constant at $10 \mathrm{~mm} / \mathrm{min}$.) using the specimen LMD 1 .

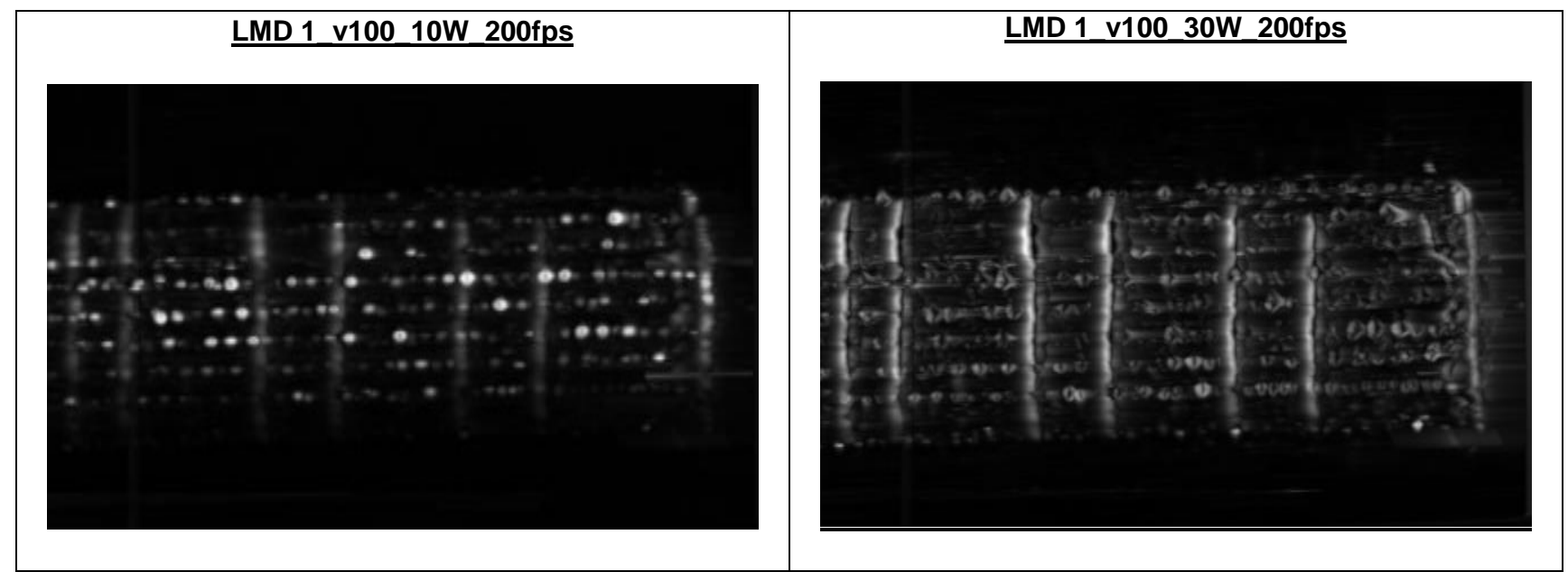

Table 2. Optimal set up parameters discussion focused on power variations (LMD).

The conclusion of studying the variation of the laser power leads to the conclusion that $30 \mathrm{~W}$ generates a clearer signal on the cracks. Once every possible combinations have been established, it is determined that the definitive 
optimal parameters for LMD are $100 \mathrm{~mm} / \mathrm{min}$. and 30W. In Table3, comparison between first and second approach results is shown for test pieces LMD 1, LMD 2 and LMD 3 when using optimal parameters.

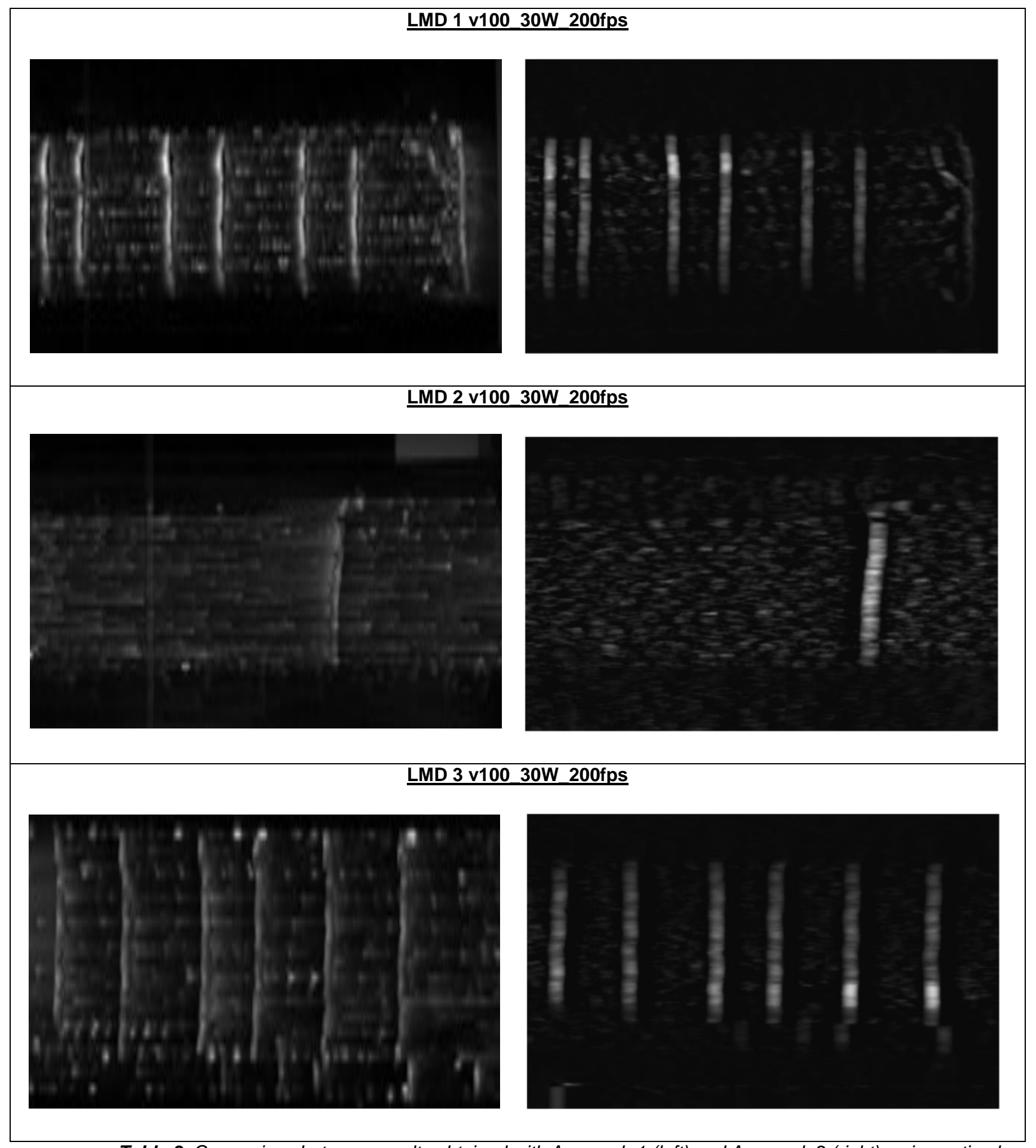

Table 3. Comparison between results obtained with Approach 1 (left) and Approach 2 (right), using optimal parameters $(100 \mathrm{~mm} / \mathrm{min}$ and $30 \mathrm{~W})$.

For the TIG welds, the definition of the final parameters is determined again by comparing the results of the detection algorithm applied to the approach 1 when fixing the power to $30 \mathrm{~W}$ and changing test speed between 50,100 and $200 \mathrm{~mm} / \mathrm{min}$ on TIG 1. 
Looking at the results shown in Table 4, the optimal speed selected is $100 \mathrm{~mm} / \mathrm{min}$. The image is a bit noisy but the SNR for $100 \mathrm{~mm} / \mathrm{min}$ is the highest. Last step is to develop tests varying laser power (30, and 50W) on TIG1 maintaining the optimal speed. These results are shown on Table 5.

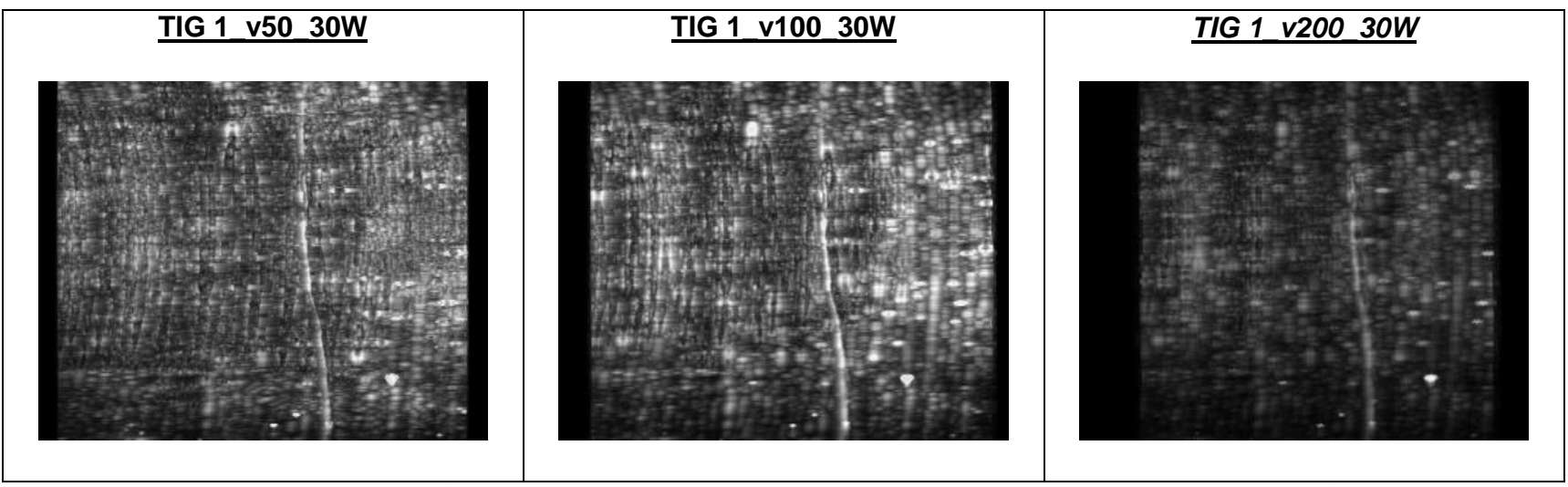

Table 4. Optimal set up parameters discussion focused on speed variations (TIG).

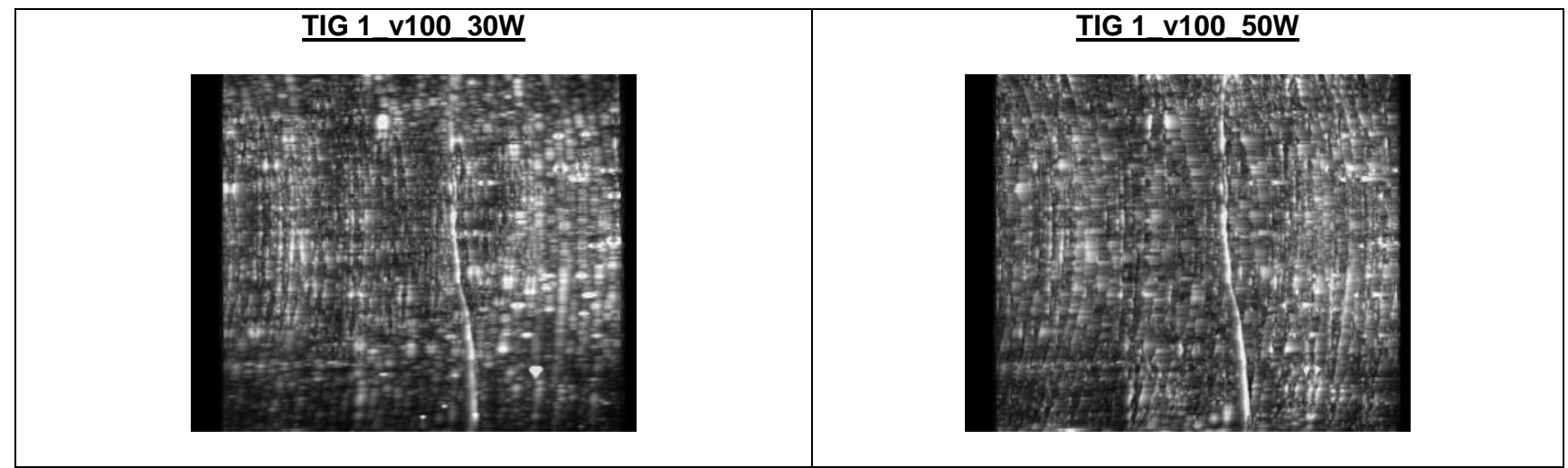

Table 5. Optimal set up parameters discussion focused on power variations (TIG).

The conclusion of studying the variation of the laser power leads to the conclusion that $50 \mathrm{~W}$ generates a higher SNR so the optimal parameters for Group Two are $100 \mathrm{~mm} / \mathrm{min}$ and 50W. In Table6, comparison between first and second approach results is shown for TIG 1 and TIG 2 when using optimal parameters.

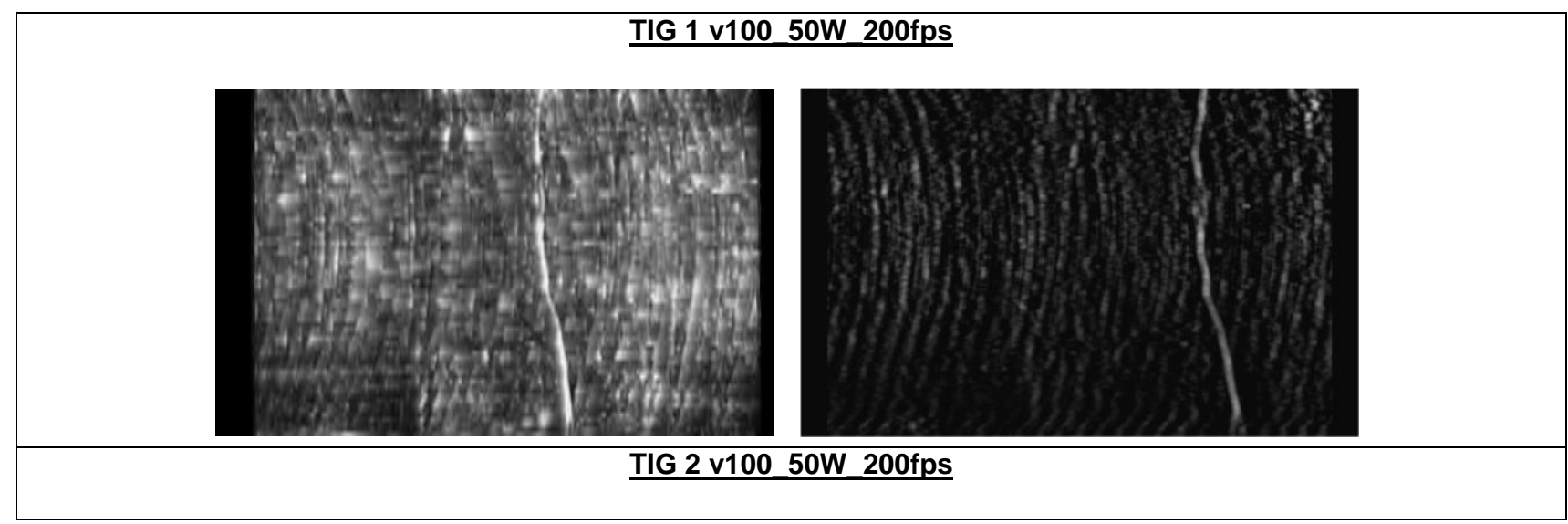




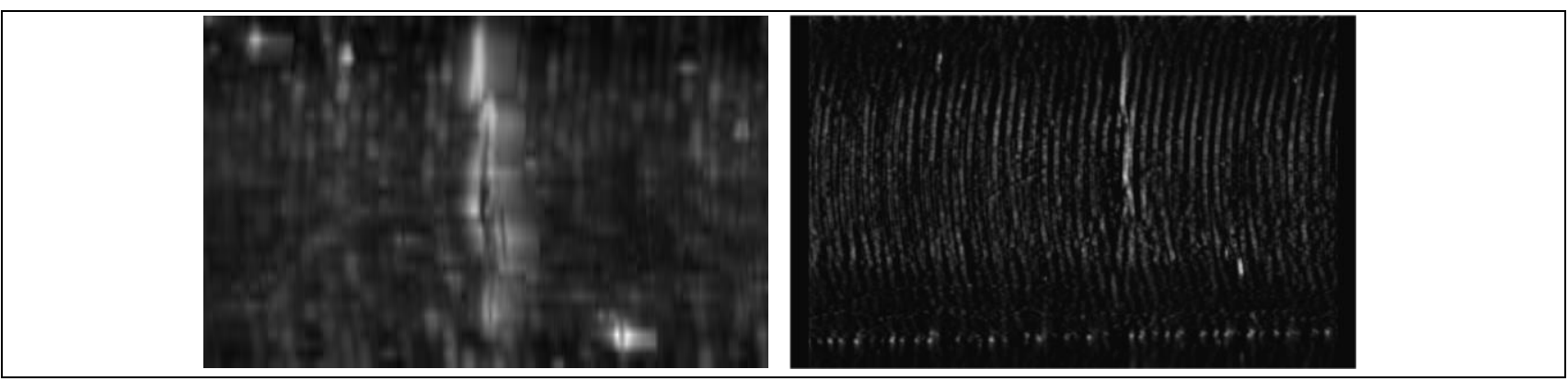

Table 6. Comparison between results obtained with Approach 1 (left) and Approach 2 (right), using optimal parameters $(100 \mathrm{~mm} / \mathrm{min}$ and $50 \mathrm{~W})$.

\section{Final conclusions}

Four LMD, looking at the results in Table 3 it can be concluded that both approaches can be used to detect the cracks appeared during the process. Taking into account the main characteristic of each approach, which are full information for the first one and reduction of this information (and the analysis time derived from it), the results obtained in Approach Two are considered better. Not only because the detection of the cracks is total, also because the algorithm is ten times faster than for approach 1.

On the second case, TIG narrow gap welding, the conclusions obtained are the same. Approach Two gives a less noisy image that permits a better crack detection. Having reduced the data to the minimum necessary on approach two allows to get rid of many false positives that damage the image in approach one.

\section{REFERENCES}

[1] Yun-Kyu An, Jinyeol Yang, Soonkyu Hwang, and Hoon Sohn. Line laser lock-in thermography for instantaneous imaging of cracks in semiconductor chips, 2015.

[2] Balageas. Theoretical and experimental applications of the flying spot camera, 1992.

[3] Patrik Broberg. Surface crack detection in welds using thermography. NDT \& E International, 57(Supplement C):69 - 73, 2013.

[4] Chiwu Bu, Qingju Tang, Yuanlin Liu, Xiangyang Jin, Zhihui Sun, and Zugen Yan. A theoretical study on vertical finite cracks detection using pulsed laser spot thermography (plst), 2015.

[5] S.E. Burrows, S. Dixon, S.G. Pickering, T. Li, and D.P. Almond. Thermographic detection of surface breaking defects using a scanning laser source. NDT \& E International, 44(7):589 - 596, 2011.

[6] E-m. Ndt\&e international 44 (2011) 216â€"225 contents lists available at sciencedirect ndt\&e international $n$ corr. 2011.

[7] C. Ibarra-Castanedo, P. Servais, A. Ziadi, M. Klein, and X. Maldague. Rita - robotized inspection by thermography and advanced processing for the inspection of aeronautical components. 1774 . 
[8] J.-C. Krapez, L. Legrandjacques, F. Lepoutre, and D. L. Balageas. Optimization of the photothermal camera for crack detection. In QIRT International Conference on Quantitative InfraRed Thermography, 1998.

[9] N. Montinaro, D. Cerniglia, and G. Pitarresi. Flying laser spot thermography technique for the nde of fibre metal laminates disbonds. Composite Structures, 171(Supplement C):63 - 76, 2017.

[10] Pech-May. Sizing the thermal resistance of vertical cracks using pulsed infrared thermography with laser spot excitation, 2016.

[11] Abdoulahad Thiam, Jean-Christophe Kneip, Eugen Cicala, Yannick Caulier, Jean-Marie Jouvard, and Simone Mattei. Modeling and optimization of open crack detection by flying spot thermography. NDT \& E International, 89(Supplement C):67 - 73, 2017. 